\title{
Lidil
}

Revue de linguistique et de didactique des langues

$52 \mid 2015$

Les pratiques artistiques dans l'apprentissage des langues

\section{Les répertoires de théâtre dans l'enseignement- apprentissage du français langue étrangère}

The Theatrical Corpus in the Teaching and Learning of French as a Foreign

Language

Ève-Marie Rollinat-Levasseur

\section{OpenEdition}

Journals

Édition électronique

URL : https://journals.openedition.org/lidil/3821

DOI : 10.4000/lidil.3821

ISSN : 1960-6052

Éditeur

UGA Éditions/Université Grenoble Alpes

Édition imprimée

Date de publication : 20 novembre 2015

Pagination : 63-81

ISBN : 978-2-84310-312-4

ISSN : $1146-6480$

Référence électronique

Ėve-Marie Rollinat-Levasseur, « Les répertoires de théâtre dans l'enseignement-apprentissage du français langue étrangère », Lidil [En ligne], 52 | 2015, mis en ligne le 01 janvier 2017, consulté le 28 juin 2022. URL : http://journals.openedition.org/lidil/3821 ; DOI : https://doi.org/10.4000/lidil.3821

(c) Lidil 


\title{
Les répertoires de théâtre dans l'enseignement-apprentissage du français langue étrangère
}

\author{
Ève-Marie Rollinat-Levasseur*
}

\begin{abstract}
RÉSUMÉ
Cet article se propose d'analyser le répertoire dramatique des textes joués en français langue étrangère (FLE) à partir de différents exemples recensés dans le monde, dans le cadre scolaire et universitaire. La recherche effectuée montre une grande variété des pratiques : sont mis en voix et en espace aussi bien des textes créés par les apprenants et/ou les enseignants, des adaptations d'œuvres de statuts divers et des pièces de théâtre. C'est la représentation que les enseignants ont de la langue française mais aussi leur connaissance du théâtre français qui influe sur les choix qu'ils font. Cependant, l'attention à leur public d'apprenants comme à celui des éventuels spectateurs des représentations données à l'issue du travail montre qu'une sorte de loi de proximité agit aussi : pratiquer le théâtre en français, ce n'est pas avoir le seul souci de la langue mais bien aussi faire du théâtre, avec toute la diversité de conceptions que cela recouvre, pour son propre plaisir et celui du public.
\end{abstract}

\section{ABSTRACT}

This article analyses the corpus of dramatic texts used in French as a Foreign Language (FLE) courses within various scholarly and university contexts around the world. The research shows a large variety of practices: texts created by students as well as by teachers are voiced and staged as are theatrical plays and adaptations of various works. The representation that the teachers have of the French language and their knowledge of French theatre influence the choices they make. But the attention they pay to their students as well as to any potential spectators demonstrates that there is a law of proximity at work as well: teaching theatre in French demands not only that one thinks about language but also about doing theatre, with all the various conceptions implicit in this act, for the pure pleasure of it and for that of the public.

* Université Sorbonne Nouvelle-Paris 3 -SPC, DILTEC EA 2288. 


\section{Introduction}

L'analyse du répertoire dramatique des textes joués en français langue étrangère (désormais FLE) permet d'approcher la façon dont la pratique théâtrale est abordée dans un contexte d'enseignement tout en donnant des renseignements sur l'enseignement-apprentissage de la langue et de la culture visé par une telle activité. Mais recenser les pratiques s'avère assez difficile. L'étude des manuels de FLE met quelques textes en lumière, sans véritablement dessiner un corpus de théâtre pour le FLE : y sont proposées de nombreuses activités de jeux de rôles, ce qui est à la limite de la pratique théâtrale ${ }^{1}$; on ne trouve que peu de textes dramatiques et ces manuels incitent rarement leurs lecteurs à se lancer dans un travail d'interprétation théâtrale (Rollinat-Levasseur, 2014²). Dans la plupart des cas, la pratique théâtrale n'entre pas dans le cursus des apprenants mais fait partie des activités récréatives et périphériques qui leur sont proposées (Alix, Lagorgette \& Rollinat-Levasseur, 2013, p. 10-11) : non obligatoires, portés par le seul désir des apprenants et des animateurs, à mi-chemin entre le théâtre amateur et la pratique théâtrale éducative, les ateliers théâtre sont alors des lieux d'expérimentation qui laissent une certaine liberté à leurs animateurs et aux apprenants, ne les inféodant pas à un objectif linguistique préalablement défini ni à un programme en littérature ou en culture française ou francophone. De ce fait, le type de jeu, le rapport aux textes destinés à être joués, les œuvres choisies découlent principalement de la culture éducative et théâtrale des enseignants-animateurs, mais aussi de leur prise en considération de ce qu'ils peuvent faire jouer aux apprenants, dans le lieu où ils se trouvent et en fonction de l'institution à laquelle ils appartiennent ainsi que du contexte social, économique et politique dans lequel ils travaillent : de là découle la grande variété du répertoire exploré. Ces pratiques restent souvent isolées. Elles connaissent toutefois une forme

1. S'ils reposent sur l'invention de situations et de personnages fictifs, les jeux de rôles sont des activités visant la production orale et n'impliquent pas de rejeu, attendu dans les jeux dramatiques, ni de recherche esthétique.

2. L'étude portait sur une quarantaine de méthodes de FLE principalement publiées en France entre 1990 et 2014, du niveau A1 à C1 (notamment : Alors, Alter ego, Bravo, Café-Crème, Campus, Écho, Édito, Escales, Ici, Le Nouvel Édito, Libre-Échange, Littérature en dialogues, Littérature progressive, Mosaïques, Métro Saint-Michel, Panorama, Scenario, Studio, Tempo, Tout va bien, Vite et bien). 
de diffusion lorsqu'elles donnent lieu à des représentations en fin de parcours, le plus souvent devant d'autres apprenants, familles ou amis, ou encore lors de festivals de théâtre français et francophones, lieux où les expériences théâtrales et linguistiques se croisent et s'enrichissent les unes les autres. Si des programmes sont produits pour ces occasions, si les enseignants ou les apprenants peuvent conserver pour eux des documents sur l'aventure théâtrale qu'ils vivent, il n'y a pas de pratique d'archivage systématique de ces entreprises. Même quand la pratique théâtrale est portée par une association ou lorsqu'elle est intégrée dans un programme de cours, il demeure ardu de recenser les programmes des œuvres jouées sur plusieurs années. Cela constitue un frein pour constituer des corpus cohérents pour une recherche dans le domaine.

C'est la raison pour laquelle cette étude repose sur des éléments divers que j'ai recueillis depuis 2008 : les communications présentées lors de colloques ou de journées d'études sur la didactique des langues, dont le FLE, notamment le colloque international de Tunis ${ }^{3}$, ceux qui se sont déroulés à l'université Stendhal-Grenoble 3 en $2010^{4}$ et en $2012^{5}$, le $8^{\mathrm{e}}$ congrès international Arts de la scène-Éducation IDEA-Paris 2013; les discussions et les conversations poursuivant les échanges; des rencontres avec des enseignants ou des apprenants ayant fait du théâtre en FLE; des articles dans des ouvrages ou des revues de didactique du FLE ou sur la pratique théâtrale scolaire et universitaire ${ }^{6}$. L'analyse des

3. «La didactique du français langue étrangère par la pratique théâtrale» organisé à l'ISSHT (Tunis) par C. Alix, Z. Benaziza, D. Lagorgette, È.-M. Rollinat-Levasseur. Une partie des interventions peuvent se trouver sous forme d'articles dans l'ouvrage issu de ce colloque et publié aux Presses de l'Université de Savoie (2013).

4. «Apprendre les langues par le théâtre», organisé conjointement par les services Culture et LANSAD de l'université Stendhal-Grenoble 3, mars 2010 : $<$ http://lansad.u-grenoble3.fr/version-francaise/recherche/journees-d-etudeet-colloques/> (consulté le 16 avril 2015).

5. «Les pratiques théâtrales dans l'apprentissage des langues : institutionnalisation et enjeux de formation au niveau européen», organisé conjointement par les services Culture et LANSAD de l'université Stendhal-Grenoble 3, en partenariat avec le GERCI, novembre 2012: <http://podcast.grenet.fr/ podcast/colloque-international-les-pratiques-theatrales-dans-lapprentissagedes-langues/> (consulté le 15 avril 2015).

6. Parmi mes nombreux informateurs, je tiens à remercier tout particulièrement Isabelle Bernard et Waël Rabadi (Université de Jordanie), Anne-Marie Havard (Festival de l'Institut français, Naples), Gaëlle Karcher (Université 
répertoires, élaborée à partir de l'ensemble de cette documentation, nous conduit tout d'abord à évoquer la question de la nature du texte choisi lui-même, à savoir l'alternative qui se pose entre choisir de travailler à partir de textes créés par les apprenants ou les enseignants dans ces activités de pratique théâtrale et décider de mettre en voix et en espace un texte dramatique préexistant. Puis, nous évoquerons les genres de textes qui semblent être privilégiés dans ce contexte spécifique d'enseignement. Enfin, nous nous pencherons sur deux cas, le corpus des textes joués en français à l'université de Princeton et celui de l'association La Ménagerie de Berlin, pour analyser comment des institutions qui intègrent le théâtre aux programmes de formation constituent leur répertoire et ce que leurs modes d'évaluation du travail théâtral en FLE révèlent de leur conception de l'apprentissage du français et des pratiques artistiques.

\section{Créations théâtrales vs théâtre de texte}

Le théâtre est souvent prôné comme une activité permettant aux apprenants de gagner en aisance dans l'expression orale. Cependant, plusieurs compétences peuvent être travaillées à travers la pratique théâtrale et la nature du texte choisi comme support est déterminante. Trois types de textes se distinguent et sont clairement représentés dans les usages attestés de la pratique du théâtre en FLE : des textes composés par les apprenants à partir de leurs improvisations qui supposent, en amont du jeu, des activités de production orale et/ou écrite; des textes créés par les apprenants et/ou par l'enseignant-animateur de l'atelier à partir de textes préexistants (contes, récits, romans, nouvelles, poèmes, scripts de films, etc.), ce qui peut impliquer la compréhension de l'écrit et susciter la créativité des apprenants pour agencer les différents matériaux retenus; des textes dramatiques anciens ou contemporains, écrits par des dramaturges pour la scène, supports de compréhension écrite à travers l'exploration du sens engagée par le jeu de l'incarnation et de l'interprétation. Le théâtre de texte ne prédomine pas : cela témoigne de la désacralisation de la littérature dans l'apprentissage-enseignement du

Stendhal-Grenoble 3), Qin Li (Université de Shanghai, Chine) Florent Masse (Université de Princeton, États-Unis), Emilia Munteanu (Université Vasile Alecsandri de Bacău, Roumanie), Marjorie Nadal (La Ménagerie, Berlin), Sylvain Tanquerel (EHESS), Daniel Mariet (FETLYF-Festival européen Théâtre lycéen francophone). 
FLE, mais aussi de ce que la pratique théâtrale en contexte éducatif est en phase avec la création scénique actuelle, laquelle voit se côtoyer des textes composés par des collectifs sur une recherche scénique à partir d'improvisations, différents types de réécritures, des mises en voix et en espace de textes non dramatiques et des mises en scène de textes de répertoire de toutes époques et du plus contemporain.

Le travail d'écriture à partir d'improvisations des apprenants est souvent lié au souci qu'a l'enseignant-animateur de leur donner la parole, y compris de leur permettre d'exprimer des sentiments personnels, des émotions ou d'évoquer leur vie, tout en les conduisant à prendre de la distance par rapport aux situations suscitées, grâce à la stylisation à laquelle conduit l'exercice. Les expériences narrées par Rosine Schautz (2013) ou Véronique Laurens (2013) sur ce type d'écriture collective avec des migrants, pour certains analphabètes, montrent comment ce travail qui part de l'écoute, de l'expression orale, peut conduire à un passage de stabilisation des textes par l'écrit (et constitue ainsi une forme d'entrée dans l'écrit) pour mener les apprenants à gagner en maitrise dans leur expression orale grâce aux nuances de la voix et du jeu qu'ils peuvent travailler : le plus souvent, ces expériences théâtrales leur permettent de mettre en scène leur aventure migratoire, la confrontation entre l'imaginaire de l'exil et la perception du pays d'accueil, le frottement entre le passé et le présent, l'attente d'un avenir, ou encore des scènes de la vie d'exilé. L'écriture d'Ariane Mnouchkine, metteur en scène notamment du Dernier Caravansérail (Théâtre du Soleil, 2003), est sans doute la figure tutélaire de cette pratique théâtrale. Mais il nous semble que s'ajoute à cela la conception d'un travail dramaturgique proche de l'art-thérapie qui s'articule étroitement à une représentation de l'apprentissage de la langue cible, à savoir qu'il n'est possible d'entrer dans la langue que lorsque les mots peuvent dire les maux et créer à partir de là un espace ouvert à l'expérimentation de l'expression en français.

Les adaptations pour la scène par les apprenants ont une forme de parenté avec l'écriture d'improvisation en ce que ce genre théâtral n'impose pas un texte préexistant mais le fait composer par les apprenants eux-mêmes (ou pour eux par l'enseignant avec le soin de prendre en compte le niveau linguistique des apprenants) à partir d'un motif, d'une intrigue, de textes ou de différents matériaux : lorsque ce sont les apprenants qui créent le texte, l'activité peut allier expression orale et expression écrite. Les répertoires que nous avons étudiés montrent en outre que ce ne sont pas toujours des pièces ni des textes français ou 
francophones qui servent de matériau pour l'écriture du spectacle. Ainsi les pièces de théâtre jouées par les étudiants de français de trois universités de Shanghai (SISU, Fudan, ECNU) lors du festival de thêâtre de l'une des universités ou de rencontres à l'Alliance française entre 2011 et 2014 ont des sources très variées : des films (qui ne sont pas écrits pour être lus) américains, tels Autant en emporte le vent ou La Reine des neiges, hongkongais, tel Dong Cheng Xi Jiu, ou encore français avec Les Choristes, le spectacle français Mozart-L'opéra rock, des romans chinois, comme Le Roi des singes de Chen-En Wu, anglais, comme Orgueil et préjudice, ou français avec Le Petit Prince, des nouvelles, telle La Parure de Maupassant, des contes (Cendrillon, BlancheNeige, Peau d'Âne...), des live-shows chinois comme If You Are the One; quelques pièces de théâtre figurent aussi à ces programmes, issues principalement du répertoire chinois, plus rarement français. Ce répertoire témoigne que le théâtre n'est pas toujours la source d'inspiration pour la pratique théâtrale : c'est le signe que le théâtre est un genre qui a perdu une part de son prestige et est relativement méconnu, du moins dans certains contextes, et cela montre que d'autres pratiques spectaculaires (cinéma, télévision, comédie musicale) sont désormais plus prégnantes dans la culture des apprenants et sans doute dans celle de leurs professeurs. Cet ensemble montre aussi que les enseignantsanimateurs ne cherchent pas à transmettre ni à imposer la culture française ou francophone par l'activité théâtrale, mais qu'ils s'attachent avant tout à trouver des supports qui puissent plaire aux apprenants et les motiver pour jouer en français. Ce qui importe donc à travers la pratique thêâtrale ainsi comprise, c'est de pratiquer le français et de jouer. Dans certains cas encore, par exemple Maboulismia joué en 2003 par la troupe francophone de l'université de Brno qui a pour source le roman Margorie d'Alexandra Berková, le choix d'adapter et de traduire une œuvre connue du pays où se fait le travail théâtral relève aussi du souci d'ajouter les activités de traduction et de réécriture à celle d'expression à l'oral, ainsi que de faire en sorte que les apprenants puissent être compris par le public local devant lequel ils vont jouer et qui, ne maitrisant pas toujours le français, peut néanmoins suivre l'intrigue et apprécier leur interprétation. Ainsi, dans ces situations où l'apprentissage de la langue française ne se fait pas dans un pays francophone ni dans le but de voyager dans un pays où cette langue permet de communiquer, pratiquer le théâtre en français conduit les apprenants à développer leur propre culture en lui donnant vie et en l'interrogeant par le détour de la scène dans une langue qui lui est étrangère, le français. 
Le montage de textes divers, poésies ou essais, comme Les Fragments d'un discours amoureux de Roland Barthes mis en scène en 2011 par Gaëlle Karcher et Aliette Lauginie avec les étudiants du Centre universitaire d'études françaises de l'université Stendhal-Grenoble 3, permet de construire un spectacle qui met en valeur les apprenants, leur donnant des passages à mettre en voix et en espace dans la mesure de leur compétences linguistiques et de leur aisance sur scène : ce travail évite le jeu de l'incarnation attendue dans les pièces de facture traditionnelle dont l'action est portée par des personnages; il se prête à une alternance de voix singulières ou chorales, ce qui conduit à n'exposer les apprenants qu'à la mesure de leurs possibilités. Ainsi, le montage, conçu pour le public auquel il est destiné, témoigne d'une conception de l'apprentissage de la langue qui associe étroitement l'expression orale et l'expression corporelle, mais pour qui le geste est avant tout esthétique, c'est-à-dire pas nécessairement redondant par rapport au texte dit.

Le théâtre de texte reste aussi prisé en FLE : la culture littéraire et théâtrale garde un poids important dans certains contextes académiques (Godard, 2015) et les pièces offrent un support préexistant qui peut séduire des enseignants qui ont une culture littéraire mais qui n'ont pas nécessairement une formation ni une pratique du théâtre, tout comme ceux qui ont une expérience de la scène. Dans le contexte du théâtre en FLE, il semble ainsi ne pas y avoir de canon, même s'il y a des tendances et que quelques textes sont souvent repris. Ainsi, comme l'ont fait apparaitre les débats lors des journées d'étude organisées à l'université Stendhal-Grenoble 3 le 5 mars 2010 sur les pratiques théâtrales dans l'apprentissage des langues à l'université et comme on peut le voir à travers l'ouvrage Didactique du français langue étrangère par la pratique théâtrale (2013), les textes privilégiés nous paraissent être: les grandes pièces qui figurent dans les programmes de littérature; les scènes métathéâtrales et celles qui reposent sur des jeux de langage parce que la réflexivité nourrit la conscientisation de l'apprentissage de la langue cible; les situations dramatiques fortes écrites dans une langue simple pour leur efficacité à la fois dramaturgique et linguistique, ce qui favorise leur inscription dans la mémoire des apprenants; le théâtre de paroles, c'est-à-dire les écritures contemporaines à l'écoute du jeu de la parole et des conversations, parce qu'elles semblent offrir une entrée dans le français contemporain. 


\section{Textes dramatiques phares}

Les textes dramatiques que nous évoquerons ici sont ceux qui nous ont paru revenir le plus fréquemment dans les répertoires considérés comme support de mise en voix et en espace. Cette activité n'est parfois qu'un contrepoint ludique et ponctuel dans l'organisation d'un cours, à l'occasion de la lecture d'un texte, mais ces textes peuvent aussi faire l'objet de projets pédagogiques allant jusqu'à leur mise en scène dans leur intégralité.

L'intérêt pour les textes de grands auteurs se justifie par leur importance dans la culture cible, mais aussi par leur force stylistique et dramaturgique. Les comédies de Molière offrent ainsi des morceaux de choix, d'autant plus que certaines pièces présentent des scènes et des passages avec des répliques brèves, ce qui permet à des apprenants de simuler des échanges vifs tout en s'amusant du comique de situation ou de répétition d'un quiproquo. La fameuse scène du Bourgeois gentilhomme où le Maitre de philosophie enseigne à Monsieur Jourdain comment articuler les voyelles (acte II, scène 4) séduit souvent les enseignants de FLE, au-delà des seuls habitués de la pratique théâtrale, comme en témoigne sa présence dans plusieurs manuels de FLE, parce que le dialogue semble servir d'appui au cours de phonétique (Anane-Bahroun \& Chebil, 2013, p. 211), que l'expression de l'admiration béate de Monsieur Jourdain et le ton docte du professeur permettent d'instaurer par l'humour une distance sur la situation d'apprentissage, ce qui peut contribuer à dédramatiser la difficulté qu'ont les apprenants à prononcer les sons vocaliques en français et conduire, par le jeu, à la surmonter. Des scènes, très simples, par exemple du Malade imaginaire, de L'École des femmes, du Médecin malgré lui peuvent être jouées dès les niveaux débutants. Pour tous les textes du répertoire, extraits ou pièces, une telle activité allie d'emblée à la pratique de l'expression orale une dimension culturelle et patrimoniale : interpréter un texte dramatique suppose de chercher à comprendre le contexte social et historique de l'action ainsi que celui de production de l'œuvre, éventuellement pour le reproduire, même de façon stylisée, à travers des choix de mises en scène, en particulier pour les décors et les costumes, ou pour s'en distinguer, pour l'actualiser et montrer comment ces textes du passé font encore sens aujourd'hui. C'est pourquoi faire jouer de telles pièces en français va souvent de pair avec une conception de jeu psychologique et la recherche d'une gestuelle redondante (Rollinat-Levasseur, 2013, p. 39-45) : comprendre et montrer ce que les personnages ressentent, 
s'exprimer à travers leurs rôles, c'est pour les apprenants le moyen de donner un ancrage corporel au texte de leurs répliques.

Pour le théâtre de Molière, s'ajoute un gain symbolique spécifique : celui d'enseigner le français en faisant pratiquer littéralement ce que d'aucuns nomment «la langue de Molière». Ainsi, la troupe de l'université d'État de Moldavie a pu prendre pour nom «Les enfants de Molière», ce qui atteste de l'importance du dramaturge dans l'imaginaire collectif des personnes qui apprennent ou enseignent le français. La présentation de cette troupe dans le bilan de la $8^{\mathrm{e}}$ édition du Festival international du théâtre francophone qui a eu lieu en Roumanie en 2013 met en valeur ce lien de façon exemplaire :

La soirée fut classique avec Les enfants de Molière de l'Université d'État de Moldavie qui ont représenté avec art et passion Le Médecin volant de Molière. En vraies disciples de Molière, les sept étudiantes nous ont fait redécouvrir le comique moliéresque et nous ont donné une leçon de lecture approfondie du texte et d'écriture scénique quasi professionnelle puisque respectant le texte et les costumes propres à la tradition classique. Les représentations ont été suivies par un débat autour de Jouer pour parler la langue de Molière ${ }^{7}$.

Dans le cadre du FLE, les mises en scène cherchent éventuellement à rendre l'esprit de la tradition classique - c'est-à-dire qu'elles en proposent une interprétation - plus qu'à reconstituer les conditions de représentation et la diction $\mathrm{du}_{\mathrm{XVII}}^{\mathrm{e}}$ siècle, comme prétend le faire aujourd'hui le théâtre baroque : de fait, l'enjeu reste, à travers ces pièces anciennes, de pratiquer le français, et la périphrase «la langue de Molière» ne désigne pas la langue du XVII ${ }^{\mathrm{e}}$ siècle mais la langue cible, ce qui ne recouvre pas exactement les usages oraux contemporains mais une langue quelque peu standardisée et idéalisée. C'est pourquoi jouer les textes du répertoire classique peut paraitre éloigné des objectifs d'un cours de langue, comme l'a souligné Marie-Line Zucchiatti (2009) à propos de l'expérience qu'elle a menée avec les étudiants de l'École supérieure de traduction et d'interprétation de Bologne (SSLMIT) : avec ses tournures archaïques, la langue de Molière s'écarte des usages du français contemporain et c'est pourquoi elle n'a pas fait monter à ses étudiants Le Bourgeois gentilhomme mais Charles Jourdain, une

7. Document à usage interne et non publié, archivé et transmis par Emilia Muteanu, Maitre de conférences à l'université Vasile Alecsandri de Bacău (Roumanie). 
adaptation qu'ils ont eux-mêmes élaborée de la comédie en cherchant à la rendre accessible aux spectateurs qui verraient leur spectacle. Le fait de pouvoir désormais facilement projeter des sous-titres sur scène permet même aux apprenants d'avoir une approche du jeu scénique distanciée par rapport au texte, soit par un travail de recherche esthétique, soit par la parodie ou en creusant l'écart entre le jeu et la parole. Dans ce cadre spécifique de l'enseignement du FLE, si la pratique d'un répertoire classique atteste un attachement parfois un peu idéaliste à la langue et à la culture françaises, elle ne relève pas pour autant d'une démarche archéologique et les mises en scène témoignent d'un double souci, celui d'une découverte du patrimoine artistique mais aussi d'une adaptation au contexte où l'atelier théâtre se déroule, ainsi qu'aux compétences linguistiques des apprenants et de leurs enseignants.

Certaines œuvres, assez récentes, ou certains auteurs reviennent fréquemment et révèlent quels critères peuvent présider à l'élection d'un texte dans le but de faire pratiquer la langue française à travers le théâtre : les œuvres du répertoire de l'absurde ou de la dérision, mais aussi le théâtre de paroles et, dans le théâtre d'aujourd'hui, des œuvres qui mettent en scène des personnages, des situations ou des conversations qui semblent exemplaires de l'usage du français contemporain. Ainsi, les pièces de Ionesco ou le théâtre de Tardieu restent souvent choisis, comme celles du dramaturge roumain d'expression française Matéi Visniec, né en 1956 et très prisé dans les pays de l'est de l'Europe. Certains textes sont assez courts, se présentant comme des saynètes, ce qui permet de les faire travailler dans leur intégralité : cela indique que les enseignants-animateurs considèrent comme important de donner l'accès à une œuvre dans son intégralité, et pas seulement à travers des extraits. Plus encore, ce type de répertoire permet d'éviter une hiérarchisation entre personnages principaux et secondaires souvent à l'œuvre dans le théâtre d'intrigue et de faciliter une répartition des rôles qui ne mette pas des apprenants plus en valeur que d'autres. Même si, pour Ionesco, Tardieu, Dubillard ou Audiberti, quelques expressions ou des mots sont un peu datés et tombés en désuétude, la langue employée s'approche du français standard, ce que vise l'enseignement-apprentissage du FLE. Ces œuvres se caractérisent pourtant par le jeu avec l'absurde, y compris dans l'usage de la langue : aussi les dialogues dramatiques ne peuvent-ils pas être pris comme des modèles de communication qu'il faudrait répéter pour pouvoir les imiter dans la langue cible puisque par excellence ils jouent des dérèglements de la langue et de la vacuité des échanges conversationnels. Ainsi, dans 
La Cantatrice chauve, l'absurde touche le lien entre les répliques, l'articulation entre les phrases et le sens même de certaines phrases; dans Un mot pour un autre, pièce la plus jouée de Tardieu, les mots attendus ont été remplacés systématiquement par d'autres sans rapport avec le contexte référentiel. Si ces pièces demeurent pourtant souvent jouées par des apprenants de français langue étrangère, c'est parce qu'aux yeux de leurs enseignants, le travail sur l'intonation, la prosodie ainsi que sur la gestuelle compte plus que la seule mémorisation des phrases (Dumontet \& Pellois, 2013) : la distance ironique correspond à la distance que l'apprenant doit avoir vis-à-vis de la langue cible pour acquérir une forme de dextérité dans son usage; le comique des textes est associé à un pouvoir désinhibant et est supposé servir à créer une atmosphère ludique et chaleureuse, propice à la mise en confiance des apprenants; plus encore, le comique lié à l'absurde impose aux apprenants une distance avec les personnages qu'ils jouent, les protégeant ainsi du piège d'une éventuelle identification psychologique qui pourrait les fragiliser par un surinvestissement personnel alors qu'ils travaillent dans le cadre d'une classe de langue ou d'un atelier. Enfin, dans ces œuvres, l'écart avec l'usage standard de la langue rend les apprenants sensibles au travail de l'écriture littéraire. Cela peut les inciter, par mimétisme, à entretenir une relation créative avec la langue qu'ils sont en train d'apprendre.

Le Nouveau Théâtre avec les œuvres de Nathalie Sarraute, puis de ses successeurs, fait aussi partie des textes de référence dans l'apprentissage du FLE presque pour des raisons inverses : focalisées sur le surgissement de la parole, ces pièces contraignent le lecteur-acteur à écouter à la fois le silence et le verbiage, à entendre les hésitations du jeu des interactions (Carlo, 2013, p. 264-267). Ces textes révèlent les dessous de la conversation, ce que Nathalie Sarraute elle-même appelait «la sous-conversation ${ }^{8}$ », c'est-à-dire l'ensemble des phénomènes sensibles et indécis qui accompagnent les interactions verbales : c'est pourquoi ils offrent à l'apprenant un support de choix pour observer le rôle des expressions figées et pour se les approprier, à condition d'explorer le sens que l'intonation vocale donne à toute prise de parole. Le début de Pour un oui ou pour un non, qui figure justement dans quelques manuels de FLE, le met en évidence à travers la dispute entre deux personnages : l'un reproche à l'autre de lui avoir dit «c'est bien...

8. Nathalie Sarraute, «Conversation et sous-conversation», dans L'Ère du soupçon, Paris, Gallimard, 1956, p. 81-122. 
ça...», sans que l'auteur donne une indication sur la façon de dire ces mots. Pour l'enseignant de FLE, la mise en voix de ce texte sert à faire découvrir aux apprenants l'importance de l'intonation dans la prise de parole, mais aussi à explorer l'étendue des variations possibles dans l'énonciation des mêmes mots : sur le plan pédagogique, l'exercice introduit donc du jeu dans la production orale, en montrant de façon évidente que parler dans une langue étrangère ne se réduit pas à produire une phrase, mais que la voix, les mimiques, les gestes signifient en même temps que les mots. Ce répertoire permet ainsi de travailler la redondance tout comme l'écart entre le geste et la parole. Textes de paroles, le théâtre de Nathalie Sarraute - comme celui de ses héritiers, Minyana, Lagarce, par exemple - ne demande pas de mise en espace avec des effets spectaculaires difficiles à créer ni exigeant des apprenants une bonne maitrise des techniques théâtrales ou encore un jeu physique : en cela ils se prêtent facilement à une interprétation dans le cadre contraint de l'espace d'une classe. Le succès de ces textes atteste la persistance du champ littéraire dans la culture éducative des enseignants qui les pratiquent, tout en montrant que ces mêmes enseignants, s'en servant comme support, ont une «vision à la fois globale et particulière de la parole en usage » (Weber, 2013, p. 259).

Une œuvre plus récente, Art de Yasmina Reza, créée à Paris en 1994 sur une scène privée, a attiré notre attention parce qu'elle est actuellement souvent choisie par des enseignants qui se lancent dans l'aventure théâtrale et qu'en cela, elle donne des indications sur ce que le répertoire théâtral leur semble apporter dans l'enseignement du français. Art est aussi la pièce contemporaine dont on peut trouver des extraits dans plusieurs manuels de FLE (Panorama 3 et Campus 4). Cette pièce montre trois amis de longue date, un dermatologue, un ingénieur et un représentant en papeterie, s'entredéchirer quand l'un d'eux, suivant son gout pour l'art moderne, a acheté une œuvre picturale blanche à un prix très élevé, ce que ne peut concevoir un autre aux gouts plus traditionnels. Il s'agit d'une œuvre brève et de facture traditionnelle : elle contient des échanges suivis entre les personnages ou des monologues et des didascalies qui indiquent les lieux où se passe l'action, les éléments essentiels du décor, certains déplacements et gestes des personnages et même certains de leurs sentiments et de leurs émotions. La mise en espace du texte est grandement guidée par les indications scéniques. C'est une pièce accessible : elle est facile à déchiffrer pour peu qu'on connaisse les conventions de présentation des dialogues dramatiques, et elle est publiée au Livre de Poche, une maison d'édition généraliste 
qui s'adresse à un public large (Théâtre, 1999). S'il y a quelques monologues longs et un peu complexes sur le plan linguistique parce que les personnages se confient, une grande partie du texte consiste en des échanges brefs, avec des répliques souvent composées d'une ou deux phrases simples. La langue est contemporaine et semble permettre de faire pratiquer le français courant mais aussi différents niveaux de langue, car on y trouve des expressions familières, voire grossières, les personnages exprimant vivement leur emportement et leurs ressentiments - mais aussi quelques termes plus recherchés, relevant d'un registre soutenu conforme à la position sociale des protagonistes. Le choix d'une œuvre de ce genre témoigne du souci que les enseignants ont de confronter les apprenants à la variation des usages de la langue et de les faire s'en imprégner, tout en restant dans un registre courant, voire soigné : mais c'est aussi, à travers une telle pièce, la langue d'une certaine bourgeoisie qui apparait comme le modèle de la langue d'apprentissage, ce qui indique, à contrario, la réticence à choisir comme texte dramatique des œuvres stylistiquement ancrées dans des usages de registres plus bas ou plus élevés, jugés plus rares, ou dans des usages caractéristiques d'une autre catégorie socio-culturelle. Enfin, si cette œuvre dramatique est jouée en classe de FLE, notamment en extraits, c'est aussi qu'elle offre matière à une réflexion et des débats pour de la production écrite ou orale assez classiques, autour de sujets sur la question du gout en matière esthétique, sur l'art «contemporain» ou sur le sens de l'amitié et le jeu des relations sociales. Un tel support permet ainsi d'associer étroitement la mise en voix et en espace du texte dramatique, c'est-à-dire d'un texte préexistant, et des activités où sont les apprenants à produire et exprimer leur pensée dans la langue cible. Mais surtout la mise en relation de ces deux types d'activité peut alors créer une dynamique de double légitimation : l'interprétation du texte dramatique rend concret le questionnement par le biais de son incarnation à travers les personnages et l'action, ce qui tisse un lien entre la question académique et la réalité du monde social; les exercices de production écrite ou orale attendus dans le cadre éducatif viennent en retour donner une légitimité au jeu dramatique.

Choisir de faire interpréter de telles œuvres dramatiques, ou du moins quelques scènes, dans le cadre de l'apprentissage du FLE montre comment les enseignants peuvent négocier l'introduction d'une activité ludique dans un contexte éducatif ou académique en se souciant de l'apport culturel des textes choisis, en permettant aux apprenants de jouer des rôles pour gagner en dextérité plus que pour s'y exprimer 
personnellement, et en articulant le contenu des textes à jouer aux activités traditionnellement pratiquées en classe de FLE.

\section{4. Étude de cas : L'Avant-Scène (Université de Princeton) et La Ménagerie (Berlin)}

Certaines institutions et certains pays intègrent la pratique théâtrale dans leurs cursus scolaires ou universitaires. Dès lors, les ateliers s'insèrent dans les programmes et doivent répondre aux objectifs induits par leur inscription dans un curriculum : ils peuvent ainsi figurer dans un programme d'art dramatique ou de formation artistique, mais aussi dans un programme de langue et culture françaises et francophones, ce qui oriente les critères d'évaluation des compétences de l'apprenant. Mais avec la pratique théâtrale et même en classe de langue, l'évaluation ne saurait s'arrêter aux seuls critères linguistiques, par exemple à la correction phonétique : la démarche de l'apprenant (les différentes étapes, l'investissement dans le jeu, la recherche du jeu, la variation et la répétition), la production en fin de parcours (la représentation finale), ainsi que son analyse réflexive, et son attitude dans le groupe et vis-àvis de ses partenaires de jeu sont des critères fréquemment identifiés comme étant à prendre en compte, ce qui permet d'associer la compétence d'interprétation de l'œuvre dramatique à celle de création ainsi qu'à celle d'appréciation des œuvres artistiques (à la fois littéraires et spectaculaires).

À l'université de Princeton aux États-Unis, les ateliers théâtre en français font partie des cours que les étudiants peuvent choisir au Département de français et d'italien dans leur cursus. Ils rassemblent ainsi des étudiants qui ont la langue et la culture françaises et francophones pour majeure ou mineure, mais aussi des étudiants qui ont le théâtre pour discipline ou encore des étudiants francophones inscrits à l'université. Ces ateliers de théâtre en français sont définis comme une combinaison de cours de langue et de cours de théâtre (l'enseignement dramatique dispensé étant conçu sur le modèle «des cours d'interprétation dispensés dans les conservatoires français»), et sont dispensés uniquement en langue française par un lecteur senior, Florent Masse, depuis $2000^{9}$. Les étudiants peuvent ainsi travailler de grandes scènes

9. Voir <www.princeton.edu/fit/resources/> (consulté le 15 avril 2015). La référence à la formation dispensée dans les conservatoires français doit s'en- 
du répertoire français, en prose et en vers, sur le modèle de celles qui sont étudiées dans les conservatoires. Par exemple, au terme de leur semestre de l'automne 2013, les étudiants de L'Avant-Scène ont proposé une présentation intitulée Fragments XIII avec des scènes tirées du Cid de Corneille, du Tartuffe, du Misanthrope, de L'École des femmes et de L'Avare de Molière, de Britannicus de Racine, du Jeu de l'amour et $d u$ hasard de Marivaux, d'Un fil à la patte de Feydeau, du Partage de midi de Claudel, et de la Princesse Maleine de Maeterlinck — cette dernière pièce se démarquant de l'ensemble, car moins connue. Cet ensemble est constitué de textes consacrés de la littérature française et de la scène théâtrale : Molière y a donc une place de choix. Il révèle le souci de faire connaitre aux apprenants, avec méthode et soin de la chronologie, les grands textes canoniques, lesquels, de ce fait, sont investis d'un pouvoir formateur, tant au niveau de la langue que du point de vue dramaturgique. La qualité des dialogues, soit par leur virtuosité et leur effet comique, soit par leur puissance émotionnelle, semble être un des critères présidant à leur élection. Cela implique que les apprenants ont à accorder une attention particulière à la maitrise de la diction et de l'expression dans la langue cible. Les étudiants de niveau avancé ont la possibilité dans un autre cours de jouer des pièces : quatre spectacles sont ainsi montés chaque année et présentés devant la communauté universitaire et la communauté francophile des environs de Princeton ${ }^{10}$. Les pièces mises en scène dans leur intégralité sont en partie celles du même répertoire que pour le travail sur des extraits. Mais L'Avant-Scène a aussi produit des œuvres modernes et contemporaines variées, comme Roberto Zucco de Koltès (2007), Ubu roi de Jarry (2008), Juste la fin du monde de Lagarce (2008), Incendies de Wajdi Mouawad (2010), Les Bonnes de Genet (2011), Les Mamelles de Tirésias d'Apollinaire (2013), L'État de siège de Camus (2013), Ma

tendre par opposition à la prégnance de l'Actor's Studio dans les cursus américains : mais plus qu'à une opposition claire entre deux traditions d'interprétation de l'héritage stanislavskien, ou entre un théâtre physique et un théâtre de paroles, cette précision vient signifier que l'atelier théâtre vise à faire jouer des textes du répertoire classique.

10. En cela, l'atelier L'Avant-Scène de Princeton perpétue une pratique du théâtre en français qui existait déjà aux tournants des $\mathrm{XIX}^{\mathrm{e}}$ et $\mathrm{XX}^{\mathrm{e}}$ siècles et qui avait suscité l'admiration et l'enthousiasme de L.-V. Gofflot, auteur du Théâtre au collège du Moyen Âge à nos jours (1907); voir RollinatLevasseur (2013, p. 41-42). 
Chambre froide de Pommerat (2014). Les textes choisis sortent ainsi du registre du plaisant qui pourrait tendre à donner l'image du théâtre comme un lieu de divertissement pour montrer la force du lien entre le théâtre et le politique, ou le théâtre et la sédition : cela montre que la formation dans une université destinée à une élite ne réduit pas la littérature en français à sa seule tradition, à un passé révolu, à des humanités qu'il conviendrait de connaitre pour se distinguer socialement.

La Ménagerie, plateforme de théâtre en français à Berlin ${ }^{11}$, est une association qui propose des interventions pédagogiques en cadre scolaire (primaire et secondaire) sous forme de projets ou d'ateliers hebdomadaires, avec la possibilité de réaliser un spectacle à partir de douze heures d'atelier. Regroupant des animateurs venus d'horizons divers (comédiens de profession ou pédagogues ayant eu une pratique théâtrale en amateurs), elle a pu mettre en scène différents textes interprétés par de jeunes Allemands âgés de 8 à 18 ans. Entre 2010 et 2013, des groupes d'apprenants de français ont ainsi pu interpréter des textes dans la langue cible avec des poésies de Prévert, Charles Cros et Tardieu, du théâtre contemporain avec Zone d'éducation prioritaire de Sonia Chiambretto (Actes Sud, 2012), Le Dieu du carnage de Yasmina Reza (Albin Michel, 2007), Les Pas perdus de Denis Bonal (Éditions théâtrales, 2000), Blues de Françoise du Chaxel (Éditions théâtrales, 2004), Pinocchio et Cendrillon de Joël Pommerat (Actes Sud, 2008 et 2012), mais aussi des pièces du $\mathrm{xx}^{\mathrm{e}}$ siècle comme Un Riche, trois pauvres de Louis Calaferte, La Cantatrice chauve et Rhinocéros de Ionesco ou Les Mariés de la tour Eiffel, livret de Jean Cocteau : ce répertoire est ainsi ouvert à la création contemporaine et en particulier à la littérature théâtrale de jeunesse et aux textes plus anciens, le plus souvent à des formes assez ludiques et volontiers burlesques. Certains de ces textes ont été joués dans leur intégralité, d'autres recoupés, certains adaptés, d'autres encore remaniés pour leur mise en voix et en espace dans le temps imparti. Cette association ne se donne donc pas pour mission d'être un conservatoire de textes littéraires français (il lui arrive aussi de monter des traductions de textes en français dans des ateliers), mais avant tout, comme le décline sa charte pédagogique, de faire découvrir et maitriser aux apprenants «leurs propres outils expressifs», de «rendre la langue française effective», «développer et maîtriser la langue française », «éprouver le plaisir théâtral», «prendre part au monde». Ce

11. Voir <www.lamenagerie.org/fr/> (consulté le 15 avril 2015). 
sont ces objectifs qui peuvent motiver l'évaluation quand l'atelier est intégré dans le cursus des élèves, que ce soit en primaire, en collège ou en lycée. Par exemple, en coordination avec La Ménagerie, la Leibnitz Schule a pu proposer à ses élèves de collège une fiche d'autoévaluation à remplir après chaque cours avec six critères : le respect des consignes, écoute et concentration; l'implication dans le groupe, moteur du projet, énergie donnée; l'être expressif avec son corps : mouvement, gestes, mimiques; l'être expressif avec sa voix : intonation et prononciation; comprendre et apprendre son texte au fur et à mesure; interaction en français avec l'intervenant. Cette démarche de type actionnel met donc en évidence le travail d'apprentissage par cœur des textes, mais l'exercice est conçu comme une façon de donner une inscription corporelle à la mémoire. Les textes littéraires sont ainsi mis au service des objectifs pédagogiques qui sont promus, ce qui ne signifie pas pour autant que la découverte y soit asservie : l'interprétation n'est pas ici considérée comme devoir de respect à la lettre des textes mais comme lieu d'expression.

\section{Conclusion}

Le répertoire dramatique travaillé dans le contexte de l'enseignementapprentissage du FLE se caractérise par sa grande variété. La représentation que les enseignants ont de la langue française et leur connaissance du théâtre français influent sur les choix qu'ils font. Mais l'attention à leur public d'apprenants comme à celui des éventuels spectateurs des représentations données à l'issue du travail montre qu'une sorte de loi de proximité agit aussi : pratiquer le théâtre en français, ce n'est pas avoir le seul souci de la langue mais bien aussi faire du théâtre, avec toute la diversité de conceptions que cela recouvre, pour son propre plaisir et celui du public. Poursuivre cette cartographie des répertoires de textes joués en français, les documenter et constituer un observatoire, par exemple sur internet, permettrait d'analyser les pratiques thêâtrales et de les faire connaitre : une telle plateforme donnerait ainsi un prolongement notable à ce qui s'effectue souvent déjà, de façon informelle et à petite échelle, lors des rencontres occasionnées dans le cadre des festivals de théâtre francophone. 


\section{RÉFÉRENCES BIBLIOGRAPHIQUES}

Alix, Christophe, Lagorgette, Dominique \& Rollinat-Levasseur, ÈveMarie (dir.). (2013). Didactique du français langue étrangère par la pratique théâtrale. Chambéry : Université de Savoie, Laboratoire LLS.

Bernard, Isabelle. (2010). Pratique théâtrale et insécurité linguistique: un exemple d'enseignement du FLE en Jordanie. Synergies Algérie, $10,225-231$.

CARÉ, Jean-Marc. (1983). Jeux de rôle : jeux drôles ou drôles de jeux. Le français dans le monde, 176, 38-42.

GodARD, Anne (dir.). (2015). La littérature dans l'enseignement du FLE. Paris : Didier.

Laurens, Véronique. (2013). Théâtre, terre d'asile. Dans C. Alix, D. Lagorgette \& È.-M. Rollinat-Levasseur (dir.), Didactique du français langue étrangère par la pratique théâtrale (p. 289-310). Chambéry : Université de Savoie, Laboratoire LLS.

Pellois, Anne. (2009). Sous-texte et parole intime : un apprentissage sans parole? Dans Théâtre et didactique : hommage à A. Brillant-Annequin, Recherches \& Travaux, 17 (hors-série), 97-104.

PierRA, Gisèle. (2006). Le corps, la voix, le texte : arts du langage en langue étrangère. Paris : L'Harmattan.

Rollinat-Levasseur, Ève-Marie. (2009). Pratiques du théâtre contemporain en classe de français langue étrangère. Dans A. Brilland-Annequin \& M. Bernanoce (dir.), Enseigner le théâtre contemporain (p. 169-179). Grenoble : CNDP.

Rollinat-Levasseur, Ève-Marie. (2013). Pratiques théâtrales en FLE: du jeu de l'imitation et des représentations dans l'apprentissage. Dans C. Alix, D. Lagorgette \& È.-M. Rollinat-Levasseur (dir.), Didactique du français langue étrangère par la pratique théâtrale (p. 31-45). Chambéry : Université de Savoie, Laboratoire LLS.

Rollinat-Levasseur, Ève-Marie. (2014). La formation au texte de théâtre dans la didactique des langues : un enjeu pour la formation. Dans M. Causa, S. Galligani \& M. Vlad (dir.), Formation et professionnalisation des enseignants de langues. Évolution des contextes, des besoins et des dispositifs (p. 115-130). Paris : Éditions Riveneuve.

Schautz, Rosine. (2013). Théâtre en situation. Dans C. Alix, D. Lagorgette \& È.-M. Rollinat-Levasseur (dir.), Didactique du français langue étrangère par la pratique théâtrale (p. 95-102). Chambéry : Université de Savoie, Laboratoire LLS.

Weber, Corinne. (2013). Pour une didactique de l'oralité : enseigner le français tel qu'il est parlé. Paris : Didier. 
Zucchiatti, Marie-Line. (2009a). Théâtre universitaire et didactique des langues étrangères. Expérience de traduction intralinguistique, construction du texte et théâtralisation. Dans M. I. Fernández García, M. L. Zucchiatti \& M. G. Biscu (dir.), L'Esperienza teatrale nella formazione dei mediatori linguistici e culturali (p. 127-139). Bologne, Italie : Bononia University Press.

ZucchiatTi, Marie-Line. (2009b). Notre théâtre est un navire. Voyage dans le temps et dans l'espace de Mystère à Marie-Galante ou Le Collier de Colomb. Dans M. I. Fernández García, M. L. Zucchiatti \& M. G. Biscu (dir.), L'Esperienza teatrale nella formazione dei mediatori linguistici e culturali (p. 223-239). Bologne, Italie : Bononia University Press. 